\title{
Psicooncología
}

ISSN: 1696-7240

\section{Consenso sobre el estándar de atención psicooncológica, complejidad psicosocial y actividad asistencial en el Instituto Catalán de Oncología}

\author{
Jorge Maté-Méndez ${ }^{1,2}$; Francisco L. Gil Moncayo ${ }^{1,2,3^{*}}$; Cristian Ochoa Arnedo ${ }^{1,4,5}$; Anna \\ Casellas-Grau ${ }^{1,6}$; Jordi Trelis Navarro ${ }^{7}$; Candela Calle Rodríguez ${ }^{8}$; Grupo Transversal de \\ Psicooncología del Instituto Catalán de Oncología ${ }^{\psi}$
}

1 Institut Català d'Oncologia. Unidad de Psicooncología. Hospital Duran i Reynals (L'Hospitalet de Llobregat, Barcelona, España).

2 Grupo de Investigación en Estrés y Salud (GIES). Facultad de Psicología. Universidad Autónoma de Barcelona (Bellaterra, Barcelona, España).

3 Coordinador, Unidad de Psicooncología. ICO-L’Hospitalet. Director Transversal de Psicooncología del ICO. Profesor Asociado Médico. Departamento de Psicologia Básica, Evolutiva y de la Educación (UAB). Observatorio de Atención Psicosocial de Cáncer del ICO.

4 Departamento de Psicología Clínica y Psicobiología, Universitat de Barcelona (Barcelona, España).

5 Institut d'Investigació Biomèdica de Bellvitge (L'Hospitalet de Llobregat, Barcelona, España).

6 Departament de Psicologia. Facultat d'Educació, Traducció i Ciències Humanes. Universitat de Vic-Universitat Central de Catalunya (Vic, Barcelona, España).

7 Director Asistencial Corporativo. Institut Català d'Oncologia (L'Hospitalet de Llobregat, Barcelona, España).

8 Directora General, Hospital Duran i Reynals, Instituto Catalán de Oncología (L’Hospitalet de Llobregat, Barcelona, España).

9 Institut Català d'Oncologia. Unidad de Psicooncología. Hospital Universitario Germans Trias i Pujol (Badalona, Barcelona, España).

10 Institut Català d'Oncologia - Consorci Sanitari Integral. Unitat de Psicooncologia ICO-CSI. Hospital Duran i Reynals y Hospital de Sant Joan Despí Moisès Broggi (Barcelona, España).

11 Institut Català d'Oncologia. Unidad de Psicooncología. Hospital Dr. Josep Trueta (Girona, España); Hospital Comarcal Sant Jaume de Calella (Barcelona, España).

12 Servicio de Psiquiatría. Hospital Universitario de Bellvitge. Institut Català d'Oncologia (L'Hospitalet de Llobregat, Barcelona, España); IDIBELL. Institut d'Investigació Biomèdica de Bellvitge (L'Hospitalet de Llobregat, Barcelona, España); Centro de Investigación Biomédica en Red en Salud Mental (CIBERSAM); Profesor Asociado Médico. Departament Ciències Clíniques. UB.

13 Institut Català d'Oncologia. Unidad de Psicooncología. Hospital Dr. Josep Trueta (Girona, España).

14 Institut Català d'Oncologia. Suport a la Dirección Asistencial Corporativa. (Hospital Duran i Reynals (L'Hospitalet de Llobregat, Barcelona, España).

15 Institut Català d'Oncologia. Unidad de Psicooncología. Hospital Dr. Josep Trueta (Girona, España); Departament de Ciències Mèdiques. Universitat de Girona (Girona, España).

16 Servicio de Psiquiatría. Hospital Universitario de Bellvitge, Institut Català d'Oncologia (ICO), Centro de Investigación Biomédica en Red Salud Mental (CIBERSAM), Departamento de Ciències Clíniques, Universitat de Barcelona (Barcelona, España), IDIBELL (L'Hospitalet de Llobregat, Barcelona, España).

$\Psi \quad$ El Grupo Transversal de Psicooncología que ha colaborado en la propuesta presentada "Consenso sobre el estándar de Atención Psicooncológica, Complejidad Psicosocial y actividad asistencial en el Instituto Catalán de Oncología", ha estado formada por las siguientes personas (orden alfabético): Milagros Bárez Villoria ${ }^{9}$; Cristina Bierge Casas ${ }^{1,10}$; Lydia Caba Vela ${ }^{11}$; Anna Casellas-Grau ${ }^{1,6}$; Sònia Fuentes Sanmartín”; Olivia García Cabrera Francisco Luis Gil Moncayo ${ }^{1,2,3}$ (Coordinador); Rosa Hernández Ribas ${ }^{12}$; Mercé Homs Romero ${ }^{13}$; Maria Lleras de Frutos ${ }^{1}$; Jun Lluch Salas ${ }^{14}$; Jorge Maté-Méndez ${ }^{1,2}$; Ana María Moral Torres ${ }^{13}$; Cristian Ochoa Arnedo ${ }^{1,4,5}$; Anna Rodriguez Morera ${ }^{15}$; Cinto Segalàs $\mathrm{Cosi}^{16}$; y Albert Sió Eroles ${ }^{1}$.

* Dirección de correspondencia Francisco L. Gil Moncayo. Unidad de Psicooncología. Instituto Catalán de Oncología. Granvia de L’Hospitalet, 199-203, 08908 L'Hospitalet de Llobregat, Barcelona (España). Email: fgil@iconcologia.net 
Resumen. Introducción: La atención psicosocial de las personas con enfermedad oncológica y la familia debe formar parte de todo modelo integral de atención que pretenda reducir el impacto vital del cáncer. Las intervenciones psicosociales han probado su eficacia en la ayuda a pacientes y familiares para afrontar las situaciones de alta complejidad psicosocial emergentes a consecuencia de un diagnóstico de cáncer. Objetivo: Definir y explicar el modelo de Atención Psicosocial del Comité Psicosocial del Instituto Catalán de Oncología (ICO) utilizando criterios de vulnerabilidad, complejidad y derivación; enmarcado y basado en los valores del ICO (centrados en las necesidades de pacientes con cáncer y sus familias). Método: El modelo que se presenta en este documento consta de cinco pilares: 1) Principios de la Práctica Psicosocial en Oncología; 2) Áreas de actuación en la Atención Psicosocial del paciente con cáncer y la familia; 3) Cribado de malestar emocional y derivación del paciente con cáncer y la familia para una atención psicooncológica específica; 4) Comité Psicosocial: (objetivos; funciones; organización; composición; disciplinas participantes; criterios de derivación y niveles de complejidad; y procedimiento); y 5) Índice de productividad. Resultados: Pacientes y familiares atendidos por el CPS mostraron mejoría estadísticamente significativa en los niveles del malestar emocional, pasando de una media inicial de 8,12/10 (EVA/ ENV) a una media 6,27/10 (EVA/ENV). Asimismo, se constata que las intervenciones derivadas del comité psicosocial redujeron el porcentaje de casos iniciales de alta complejidad, pasando de un $69,3 \%$ a un $49,3 \%$. Conclusiones: El abordaje de la complejidad psicosocial mediante un modelo basado en criterios multi e interdisciplinarios consensuados ayuda en la toma de decisiones sobre las acciones a seguir y en la mejora del malestar emocional y complejidad de los pacientes y la familia. Palabras clave: Cáncer; psicooncología; malestar emocional; profesionales sanitarios; factores psicosociales; complejidad.

\section{[en] Consensus on the standard of psycho-oncological care, psychosocial complexity and care activity at the Catalan Institute of Oncology}

Abstract. Introduction: Psychosocial care for cancer patients and their families should be part
of all comprehensive model of care that aims to reduce the life impact of cancer. Psychosocial
interventions have proven to be effective in helping patients and families to cope with the highly
complex psychosocial situations that arise as a result of a cancer diagnosis. Aim: To define and
explain the model of Psychosocial Care in the Psychosocial Committee of the Catalan Institute of
Oncology (ICO) using criteria of vulnerability, complexity and referral; framed and based on the
values of the ICO (focused on patients and family's needs). Method: The model presented in this
document consists of five pillars: 1) Principles of Psychosocial Practice in Oncology; 2) Areas of
action in the Psychosocial Care of the cancer patient and family; 3) Screening for emotional distress
and referral of the cancer patient and family for specific psycho-oncological care; 4) Psychosocial
Committee: (objectives; functions; organization; composition; participating disciplines; referral
criteria and levels of complexity; and procedure); and 5) Productivity index. Results: Patients and
relatives who were attended by the CPS showed statistically significant improvement in the levels
of emotional distress, going from an initial mean of 8.12 / 10 (VAS / ENV) to a mean of 6.27 / 10
(VAS / ENV). It also shows that the interventions derived from the psychosocial committee reduced
the percentage of initial cases of high complexity, from $69.3 \%$ to $49.3 \%$. Conclusions: Addressing
psychosocial complexity by means of a model based on consensual multi- and interdisciplinary
criteria helps in making decisions about the actions to be taken and in improving the emotional
distress and complexity of the patients and the family. Keywords: Cancer; psycho-oncology; emotional distress; health professionals; psychosocial factors; complexity.

Sumario: 1. Introducción 2. Principios de la Práctica Psicosocial en Oncología 3. Áreas de actuación en la Atención Psicosocial del paciente oncológico y la familia 4. Cribado de malestar emocional y derivación de la persona con enfermedad oncológica y su familia para una atención psicooncológica específica. 4.1. Criterios de complejidad psicosocial 5. Comité Psicosocial (CPS) 5.1. Objetivos del CPS 5.2. Funciones y ámbitos de actuación del CPS 5.3. Organización del CPS y circuitos de 
la Atención Psicosocial 5.4. Composición del Comité Psicosocial 5.5. Funciones y Ámbitos de las diferentes disciplinas participantes en el Comité Psicosocial 5.6. Criterios de Derivación y Niveles de Complejidad en el CPS 5.7. Procedimiento 6. Índice de productividad de Psicooncología 7. Conclusiones 8. Agradecimientos 9. Referencias bibliográficas

Como citar: Maté-Méndez J, Gil Moncayo FJ, Ochoa Arnedo C, Casellas-Grau A, Trelis Navarro J, Calle Rodríguez C, Grupo Transversal de Psicooncología del Instituto Catalán de Oncología. Consenso sobre el estándar de atención psicooncológica, complejidad psicosocial y actividad asistencial en el Instituto Catalán de Oncología. Psicooncología 2021; 18: 11-36. doi: 10.5209/psic.74530

\section{Introducción}

El presente documento muestra el modelo de Atención Psicosocial de la Unidad de Psicooncología del Instituto Catalán de Oncología (ICO). Describe los criterios de vulnerabilidad, complejidad y derivación atendidos por el comité psicosocial (CPS) que están enmarcados en los valores propuestos por la Dirección del ICO centrados en las necesidades de pacientes y sus familias: 1) Pensamos como pacientes; 2) Calidad y Calidez; 3) Decisiones con conocimiento; 4) Equipos a tu lado; 5) Investigación e innovación para mejorar el futuro. Se exponen los resultados a partir de las reuniones realizadas en el marco de la Dirección Transversal de Psicooncología del Instituto Catalán de Oncología (ICO) para el consenso sobre cuál debe ser el estándar de atención psicosocial de pacientes y familiares con cáncer, en cualquiera de las fases de su enfermedad. Asimismo, el presente artículo pretende ser una propuesta que aporte algunas herramientas que resulten útiles para otros equipos y en otras comunidades, adaptándolas a su realidad, para la atención de pacientes con cáncer y su familia.

La atención psicooncológica en los hospitales tiene un carácter estructural; debe formar parte de la estructura asistencial de centro hospitalario, considerando al profesional de la Psicooncología un/a facultativo/a más del centro donde trabaje. La atención psicosocial de las personas diagnosticadas de cáncer también debe formar parte de cualquier modelo integral de atención que pretenda reducir el impacto vital del cáncer. Las intervenciones psicosociales han probado su eficacia en la ayuda a pacientes y familiares para afrontar las situaciones complejas y potencialmente desbordantes que aparecen como consecuencia de un diagnóstico de cáncer y los tratamientos. Estas intervenciones han demostrado no solo ser efectivas para una mejor atención psicosocial, sino también eficientes en el análisis coste-efectividad ${ }^{(1)}$.

La complejidad psicosocial en cáncer se caracteriza por ser multifactorial y multidimensional, es decir, se compone de múltiples elementos biospsicosociales, espirituales y éticos que se interrelacionan en situaciones cambiantes y evolutivas. Hasta fechas muy recientes, nuestro país carecía de guías o Estándares de Atención Psicosocial similares a las de otros países ${ }^{(2,3)}$, pero fruto de un esfuerzo promovido desde instituciones como el ICO que aúna la experiencia clínica multidisciplinaria acumulada, se ha podido ofrecer una primera propuesta tanto de la definición de la complejidad psicosocial en oncología ${ }^{(4)}$ como del abordaje de la misma ${ }^{(5)}$. El Hospital Duran y Reynals del Instituto Catalán de Oncología (ICO-DiR) fue pionero en este ámbito, disponiendo de la primera propuesta de Comité Psicosocial multidisciplinario creado en el 2002 y con la elaboración de una guía básica de atención psicosocial en Oncología ${ }^{(6)}$. 
Recientemente y en nuestro país, Casellas-Grau et al., (2020)(4) han presentado la definición de complejidad psicosocial en pacientes con cáncer. Esta definición se construyó en consenso de personas expertas realizado mediante metodología Delphi y ha permitido la elaboración de una lista de indicadores pertenecientes a las cuatro dimensiones de la complejidad psicosocial -médica-física, socioamiliar, psicoemocional y espiritual-. Esta lista, tras un proceso de validación, facilita a los y las profesionales la identificación de pacientes con alta complejidad que requieren una atención prioritaria.

Padecer cáncer y los tratamientos asociados tiene un gran impacto tanto en la calidad de vida de las personas con cáncer y sus familiares, y cuidadores/as. Hasta en un 30\%-45\% de pacientes con cáncer y supervivientes refieren malestar emocional asociado $^{(7,8)}$, pudiendo presentar trastornos psicopatológicos significativos (ansiedad, depresión y trastornos adaptivos $)^{(9-11)}$. Estos trastornos requieren una atención psicosocial especializada en la medida que repercuten negativamente a nivel clínico, en el cumplimiento del tratamiento, la supervivencia y la calidad de vida ${ }^{(12)}$.

Las reacciones emocionales e implicaciones psicosociales en pacientes con diagnósticos oncológicos está bien documentada, a destacar la dificultades y trastornos de adaptación, clínica afectiva depresiva y ansiosa como miedo, tristeza, impotencia y desesperanza, depresión, trastornos por estrés postraumático, y conflictos familiares o crisis existenciales ${ }^{(13)}$. De acuerdo con McCarter $(2015)^{(14)}$, el malestar emocional que presentan las personas con cáncer (emotional distress) no siempre es reconocido por los/as profesionales que los atienden, pese a que se estima que el malestar emocional está presente hasta en un $45 \%$ de los pacientes en algún momento de la enfermedad.

La complejidad psicosocial está condicionada en gran parte por la presencia de psicopatología mayor. Algunos estudios muestran que la prevalencia de trastornos mentales en cáncer puede oscilar entre el 9,8\% y el 38,2\%(15-18). En el estudio realizado por Mitchell et al., (2011) $)^{(18)}$, la frecuencia fue del 38,2\% cuando se combinaban diferentes diagnósticos psicopatológicos (ej, depresión, distimia, ansiedad y trastornos adaptativos). Cuando las personas presentan enfermedad más avanzada, la complejidad psicosocial impresiona que aumenta, ya que la prevalencia de sufrimiento emocional es mayor hasta en el 50\% de pacientes con cáncer avanzado, y se estima que entre un $35 \%$ y un $50 \%$ de pacientes en cuidados paliativos pueden presentar algún trastorno afectivo durante el curso de su enfermedad ${ }^{(17)}$.

Algunos estudios y en la línea de lo comentado anteriormente, han evidenciado que la atención oncológica multidisciplinaria pueden influir en la mortalidad y supervivencia. En el estudio de Galán et al. $(2015)^{(19)}$ con pacientes con cáncer de esófago y de la unión esofagogástrica, se describe cómo un enfoque multidisciplinario dio lugar a una reducción significativa de la mortalidad quirúrgica, y un aumento significativo de la supervivencia. Otros estudios también evidencian que la atención multidisciplinaria por excelencia que ofrecen equipos de Cuidados Paliativos, facilitan no solo un mejor control sintomático sino también el afrontamiento de la enfermedad. Como ejemplo en el estudio clínico aleatorizado de Temel et al. (2010) ${ }^{(20)}$, se mostró que aquellas personas con cáncer avanzado que recibieron atención paliativa desde el principio conjuntamente con el tratamiento habitual para su cáncer refirieron no solo tener mejor calidad de vida y estado de ánimo que aquellas que no recibieron cuidados paliativos desde el principio, sino que también reportaron tener mejor capacidad para afrontar su enfermedad y aumentaron su supervivencia en dos meses. 
"El Instituto Catalán de Oncología (ICO) nació entendiendo que la calidad es uno de sus fundamentos, no tan solo como una obligación sino como una convicción y necesidad de hacer mejor las cosas para alcanzar nuestra misión, que es reducir el impacto del cáncer. Solamente si este espíritu se impregna a todos los niveles de la organización se convertirá en uno de sus objetivos estratégicos"(21).

Consideramos que la mejor manera para atender a la persona afectada de cáncer y su familia es desde la multi e interdisciplinariedad, de acuerdo con la Declaración política sobre la atención multidisciplinar del cáncer del Grupo de Consenso de la Asociación Europea contra el Cáncer ${ }^{(22)}$; y la Declaración Institucional para el Desarrollo de la Atención Multidisciplinar en Cáncer en España ${ }^{(23)}$ :

"El ámbito de la atención oncológica se caracteriza por una elevada complejidad clínica (tratamientos cada vez más multidisciplinarios y específicos) y un número creciente de casos. Este entorno ha propiciado y fomentado la especialización compartimentada por enfermedad tumoral entre las distintas especialidades y profesionales, lo que a su vez ha puesto de manifiesto la necesidad de reagrupar a todos los profesionales bajo un único paraguas organizativo que asegure una visión global del paciente" (p587) ${ }^{1}$.

Para atender la complejidad psicosocial es fundamental disponer de una definición consensuada de la misma que permita enfocar la atención psicosocial de acuerdo con un guía derivada de esta definición. Estudios recientes han solicitado definiciones ampliamente consensuadas de la complejidad psicosocial para aumentar la eficacia de la atención y establecer prácticas adecuadas en los hospitales ${ }^{(24)}$. Tuca et al. (2018) ${ }^{(25)}$, utilizando un modelo predictivo de complejidad en los cuidados paliativos avanzados y centrándose en pacientes con cáncer avanzado, hallaron diferencias significativas entre grupos al comparar la complejidad alta-media-baja para variables sociodemográficas y médicas. En su estudio, aquellos/as pacientes que mostraban una alta complejidad eran más jóvenes, tenían un mayor número de síntomas y más intensos; asimismo presentaban más factores de riesgo social/ familiar, más problemas existenciales/éticos, y mayores dificultades tanto de rendimiento como funcionales.

Tras el trabajo de consenso realizado por el mismo grupo de trabajo que suscriben este documento, la definición de Complejidad Psicosocial en el cáncer resultante del estudio Delphi liderado por Casellas-Grau et al. (2020) $)^{(4)}$ es la siguiente:

"La complejidad psicosocial en los pacientes con cáncer es un concepto multidimensional en el que el paciente experimenta una alta sensación de amenaza, lo que provoca dificultades para afrontar la enfermedad, especialmente cuando ésta tiene un pronóstico malo o incierto. Estas dificultades generan malestar emocional en el paciente, manifestadas a partir de reacciones traumáticas poco adaptativas, como síntomas de ansiedad y depresión severas, especialmente en pacientes con historia de tentativas de suicidio. Las dificultades sociales, como un bajo soporte o un riesgo de exclusión social agravan la complejidad psicosocial del paciente. Hace falta que el equipo asistencial lleve a cabo un correcto seguimiento del caso, dado que un manejo inadecuado por parte del equipo es un elemento más de complejidad psicosocial en pacientes con cáncer” (p9)ํ. 
Teniendo en cuenta todo lo anterior, es importante tener presentes de nuevo las recomendaciones sugeridas por la European Guide for Quality National Cancer Control Programmes ${ }^{(6,27)}$ para una adecuada atención psicooncológica:

1) formación de profesionales sobre aspectos psicosociales en cáncer;

2) inclusión rutinaria del cribado del malestar emocional como sexto signo vital en pacientes con cáncer;

3) utilización de tratamientos basados en la evidencia para el manejo de síntomas y necesidades psicosociales a través del cribado del malestar emocional (emotional distress);

4) desarrollo de criterios de práctica clínica en los servicios de psicooncología;

5) implementación e integración de programas de atención psicooncológica en equipos multidisciplinarios en cáncer;

6) compromiso de las instituciones que proveen atención de atención oncológica para la inclusión de la atención psicosocial.

A modo de conclusión y tras la literatura revisada para la realización de este documento nos sugiere que (adaptado de Maté et al., 2019)(28): 1) Muchas personas con cáncer pueden sufrir durante el proceso de su enfermedad un malestar emocional significativo que pueden constituir en algunos casos un trastorno mental recogido en los manuales diagnósticos; 2) La capacidad de afrontamiento de la persona puede verse comprometida; 3) En bastantes ocasiones el malestar emocional es infradiagnosticado y, por tanto, infratratado; 4) Un malestar significativo y un afrontamiento inadecuado o deficiente repercutirá negativamente sobre la calidad de vida y el bienestar del paciente, y en la mayoría de las ocasiones, sobre sus cuidadores/as principales; 5) Las personas con diagnósticos oncológicos (y sus familiares) en este contexto se quejarán más de los síntomas (tanto en cantidad como en intensidad) y serán más demandantes de atención; 6) Pese a que la atención psicosocial de las personas diagnosticadas de cáncer forma parte de cualquier modelo integral para reducir el impacto vital del cáncer, hay escasez de guías específica en nuestro país; 7) Para atender la complejidad psicosocial es fundamental disponer de una definición consensuada de la misma; 8) Hay algunas razones importantes a favor de realizar una identificación temprana de pacientes con elevada vulnerabilidad psicosocial, ya que permite: a) una derivación adecuada (profesionales de la psicología clínica, de la psiquiatría y del trabajo social), siempre de acuerdo con la voluntad de la persona que padece y en previsión de un encarnizamiento psicosocial yatrogénico; b) una atención precoz especializada; c) facilitar una mejor adaptación al proceso; d) un menor "consumo" de recursos sanitarios; e) una optimización de la efectividad de los tratamientos; f) una mejora de la calidad de vida; g) mejor tolerancia a los tratamientos; y h) mayor supervivencia.

\section{Principios de la Práctica Psicosocial en Oncología}

La atención psicosocial que resumimos en este documento de consenso está basada en los principios básicos sobre la práctica psicosocial en oncología (Tabla 1). 
Tabla 1. Principios de la práctica psicosocial en oncología (adaptado de Gil et al., 2004) ${ }^{(6)}$.

\section{PRINCIPIOS DE LA PRÁCTICA PSICOSOCIAL EN ONCOLOGÍA}

1. La atención psicosocial en Oncología forma parte del cuidado integral de las personas enfermas de cáncer.

2. La persona enferma y la familia es la unidad básica de atención psicosocial en cáncer.

3. Las personas con cáncer y sus familias deben ser informadas y tener acceso a los servicios de atención psicosocial que la institución dispone.

4. Los/las profesionales en su intervención deben respetar la dignidad e integridad del individuo.

5. La atención psicosocial a paciente y familia debe estar disponible a lo largo de todas las fases o estadios de la enfermedad oncológica.

6. Los aspectos psicológicos y sociales deben ser valorados por el equipo asistencial a través de instrumentos de evaluación breves e indicadores validados de riesgovulnerabilidad y necesidades psicosociales.

7. Las personas que componen los equipos profesionales interdisciplinarios deben respetar la experiencia y el conocimiento que cada una de ellas posee.

8. La práctica psicosocial debe estar basada en la evidencia y sujeta a posibles cambios para aumentar su efectividad.

9. La formación continuada e investigación por parte de las personas que componen el equipo psicosocial son dos componentes esenciales para la práctica asistencial.

\section{3. Áreas de actuación en la Atención Psicosocial del de la persona con enfermedad oncológica $^{2}$ y su familia}

Definimos cuatro áreas de actuación fundamentales:

- Asistencial: dirigida a paciente y familia, con el objetivo de ayudar a asimilar, integrar y normalizar los cambios producidos por la enfermedad.

- Docente: a través de su supervisión a residentes y estudiantes, así como su colaboración con programas docentes de formación continuada, grado y post-grado a todo profesional sanitario.

- Investigación: generando conocimiento y evidencia de la eficacia de las intervenciones psicológicas y psicoterapéuticas, así procurando por su divulgación.

- Gestión: priorizando la atención a pacientes o familiares que presenten alta complejidad psicosocial.

2 Por persona con enfermedad oncológica entendemos en este documento paciente con diagnóstico oncológico y hematológico. 


\section{Cribado de malestar emocional y derivación de la persona con enfermedad oncológica y su familia para una atención psicooncológica específica}

Toda derivación a Psicooncología ha de estar argumentada en función del nivel de malestar emocional y de complejidad psicosocial (Figura 1). Asimismo, debe haber un foco oncológico en toda derivación, tanto en la persona con cáncer como en su familiar. Es decir, si la razón de atención psicológica o psiquiátrica no está asociada a la enfermedad oncológica o sus tratamientos, deberá dirigirse a otro recurso o dispositivo de salud mental, público o privado.

Figura 1. Circuito de Evaluación y Derivación de casos al CPS por Complejidad Psicosocial (modificado de Gil et al., 2020) $)^{(5)}$.

\begin{tabular}{|c|}
\hline $\begin{array}{c}\text { Criterio clínico del profesional sanitario sobre la } \\
\text { Idoneidad de la Valoración Psicosocial }\end{array}$ \\
\hline
\end{tabular}

Confirmación Screening Psicosocial POSITIVO. Cumple:

1. EVA malestar emocional $>5$

2. Identificación de problemas psicosociales

REFERENTE PSICOSOCIAL DEL RECURSO ASISTENCIAL ¿Presenta complejidad psicosocial? Valorar si:

1. Cumple criterios de vulnerabilidad y riesgo psicosocial

2. Se identifica nivel de complejidad psicosocial para remitir al CPS

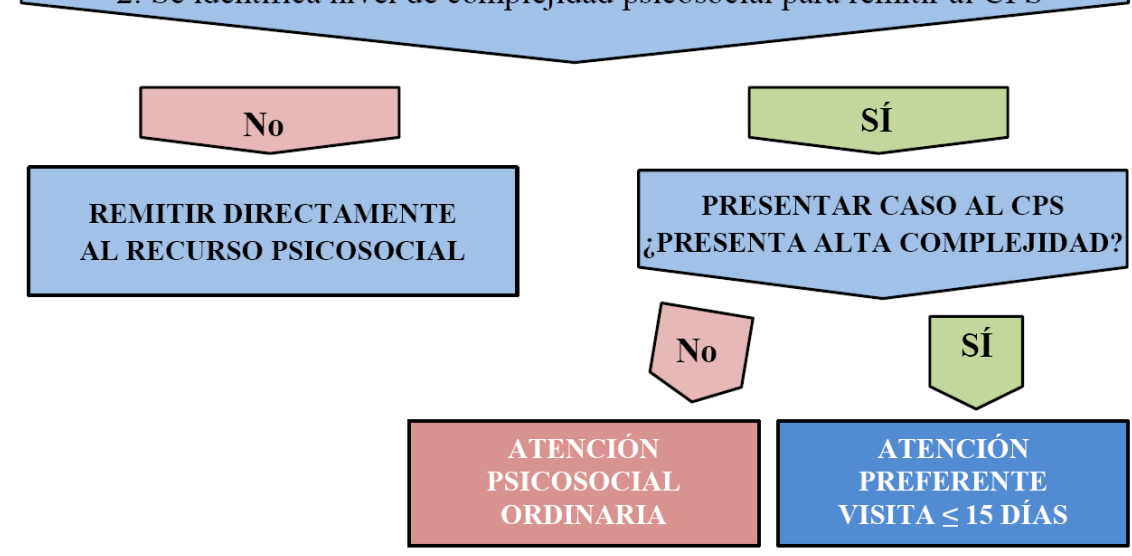

La intervención psicooncológica tiene como objetivo ayudar al a las personas con cáncer y la familia a asimilar e integrar la experiencia de la enfermedad, en cualquiera de sus fases o estadios. Hablamos de poder generar narrativas adaptativas, donde la centralidad del discurso de la persona no sea solo su enfermedad. Cuando la intervención logra que la centralidad del pensamiento o discurso no sea únicamente la enfermedad o los tratamientos (foco oncológico), volviendo a realizar su vida 
personal, adaptándose a los cambios que hayan ocurrido o estén sucediendo, podemos concluir que la intervención ha sido eficaz, que que la persona recobra su funcionalidad, y con ello finalizamos la atención prestada.

Asimismo, junto a la desaparición del foco oncológico, en la demanda de ayuda de pacientes o familiares, debe acompañarse con la remisión de síntomas afectivos ansiosos, depresivos y/o adaptativos, como muestra de reducción o desaparición del malestar emocional, como criterios a seguir para dar de alta a la persona de nuestra prestación asistencial.

En el caso de que ela persona se encuentre en una fase avanzada-terminal de la enfermedad, mantendremos la atención psicológica hasta la muerte, con el objetivo de proveer, conjuntamente con todo el equipo asistencial y en la medida de lo posible, una muerte en paz. La atención psicológica irá dirigida a la persona con diagnóstico oncológico y a la familia. Tras el fallecimiento, se informará a la familia de los recursos de atención al duelo que disponga la institución, u otros dispositivos de la comunidad.

Los criterios de derivación desde consultas externas o planta de hospitalización a Psicooncología estará determinada por: 1) el grado de malestar emocional; y 2) el nivel de complejidad psicosocial (Figura 1).

Será el/la profesionales de medicina, enfermería, trabajo social o psiquiatría quien realice la interconsulta a Psicooncología, teniendo en cuenta los criterios de derivación comentados anteriormente (grado de malestar emocional y nivel complejidad psicosocial). Asimismo, en la interconsulta se deben exponer los motivos de la derivación y además debe ser consultada y consensuada previamente con paciente o familiar a valorar.

La evaluación psicosocial que recomendamos a los/las profesionales de enfermería, medicina y trabajo social consiste en la realización de una exploración breve especialmente dirigida a la detección del malestar emocional y complejidad psicosocial. La aproximación al conocimiento del nivel de complejidad psicosocial de la persona con cáncer y/o familia es factible si prestamos atención a los indicadores de vulnerabilidad y riesgo psicosocial facilitados a modo de guía en los Anexos 1 y 2 del Acta del CPS (Figuras 4 y 5). De acuerdo con estos criterios clínicos, los/las profesionales del ámbito sanitario tiene a su disposición información sobre el circuito de evaluación y derivación de casos al CPS así como información más ampliada si la necesitan para la presentación del caso, discusión de casos y priorización en la atención (Figura 1); y Anexos I y II del Acta del CPS (Figuras 4 y 5).

Pacientes y familiares en seguimiento ambulatorio (consultas externas) que se consideren de complejidad, se presentarán por la persona solicitante (profesional de la medicina, enfermería (gestora de casos), trabajo social, psicooncología o psiquiatría) en el CPS con el fin de priorizar su visita antes de 15 días (indicador de calidad asistencial del ICO). Las interconsultas de planta a Psicooncología deben ser atendidas antes de 48 horas (indicador de calidad asistencial), y presentadas en la sesión clínica o de objetivos semanal de la planta de hospitalización para su discusión y abordaje interdisciplinar.

\subsection{Criterios de complejidad psicosocial}

Hasta julio de 2018, la complejidad psicosocial atendida por la Unidad de Psicooncología (PSO) se ordenaban en tres niveles (alta, media o baja complejidad), 
de acuerdo con criterios clínicos consensuados, aunque sin evidencia científica. En función de esta clasificación, se priorizaba la atención psicosocial, es decir, a mayor complejidad, una atención más temprana y/o especializada.

Desde julio de 2018, esta clasificación de la complejidad psicosocial realizada con criterios clínicos aunque subjetivos fue puesta a prueba mediante un estudio Delphi realizado por la PSO del $\mathrm{ICO}^{(5)}$. A partir de entonces, los casos son identificados como "alta o no alta complejidad", y es en el CPS donde tras la discusión del caso clínico, puede ser priorizada su atención en función de la misma. Por tanto, las personas que presentan altos niveles de complejidad psicosocial son preferentes en su atención y se visitan en menos de dos semanas desde la fecha que se presentaron en el comité.

\section{Comité Psicosocial (CPS)}

\subsection{Objetivos del CPS}

El CPS es el órgano que guía la atención psicosocial en Oncología, de acuerdo con unos principios de la práctica psicosocial (Tabla 1), para facilitar una mayor homogeneidad y distribución equitativa de la atención psicosocial que ha de recibir el la persona con diagnóstico oncológico y su familia. Esta guía de atención psicosocial, también busca el objetivo de servir como modelo de referencia para la atención a paciente y familia por parte de los profesionales responsables de esta asistencia (profesionales de la psicología clínica, trabajo social, enfermería de práctica avanzada y psiquiatría, y en algunos casos, de la medicina). Asimismo es un instrumento de apoyo profesionales con implicación en tareas asistenciales (enfermería y medicina) en relación a la derivación de las personas a los diferentes recursos y dispositivos de la comunidad.

\subsection{Funciones y ámbitos de actuación del CPS}

El CPS tiene unas funciones y ámbitos de actuación específicas (Figura 2), y sus principales objetivos son la valoración, derivación y atención continuada de la complejidad psicosocial en a persona con diagnóstico oncológico y la familia, tanto a nivel ambulatorio como de ingreso hospitalario. De la misma manera que en el ámbito de hospitalización se realizan de manera habitual reuniones de objetivos interdisciplinarias donde participan equipos psicosociales, el CPS se encarga de la coordinación y continuidad de la atención psicosocial a nivel ambulatorio tras el alta de la persona ingresada. Los/as referentes psicosociales de los diferentes recursos asistenciales también presentarán los casos al CPS cuando se considere oportuno mantener una continuidad en la atención psicosocial entre el ámbito ambulatorio y de ingreso hospitalario, entendiendo la continuidad en ambos sentidos.

Los miembros del CPS, desde un punto de vista interdisciplinario, presentarán los casos de primera visita o de seguimiento psicosocial (segundas visitas) con un nivel de complejidad identificado previamente. La discusión de los casos presentados favorecerá un mejor manejo psicosocial, una correcta derivación al especialista, y con esto, una mejor gestión de los recursos asistenciales a nivel psicosocial. 
Figura 2. Funciones y Ámbitos de Actuación del Comité Psicosocial (CPS),

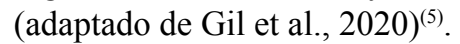

\begin{tabular}{|c|c|}
\hline FUNCIONES & ÁMBITOS DE ACTUACIÓN \\
\hline $\begin{array}{c}\text { Evaluación, Diagnóstico } \\
\text { y Toma de Decisiones }\end{array}$ & Atención Ambulatoria \\
\hline $\begin{array}{c}\text { Seguimiento y Atención } \\
\text { Continuada }\end{array}$ & Atención Hospitalaria \\
\hline $\begin{array}{c}\text { Coordinación y } \\
\text { Trabajo en Red }\end{array}$ & Comunidad \\
\hline
\end{tabular}

\subsection{Organización del CPS y circuitos de la Atención Psicosocial}

Entre las funciones del CPS destacamos la de reunir a diferentes profesionales que se implican en la asistencia psicosocial en un espacio común (presencial y/o virtual) para ofrecer una atención integral, coordinada, completa y de calidad. No es únicamente una combinación de profesionales del ámbito de la sanidad, sino una posibilidad constante de estar en contacto, solucionar cuestiones diversas vinculadas a la persona con cáncer y su familia, optimizando y agilizando procesos.

Los casos clínicos (paciente o familiar), generalmente serán derivados al CPS por parte de sus miembros pero la posibilidad de presentar casos está abierta al resto del personal sanitario asistencial. Los/as profesionales de enfermería y medicina disponen de los indicadores de derivación (cribado y complejidad) para evaluar la idoneidad de presentación de los casos al CPS. El/la profesional que lo considere oportuno podrá consultar el caso a presentar al CPS con el referente psicosocial de su recurso asistencial, para valorar si la presentación es procedente y decidir quién de los dos lo presentará. Cada servicio acordará con el coordinador del CPS quién tiene que ser su referente psicosocial. La Figura 1 presentada anteriormente resume los pasos a realizar para la presentación de un caso al CPS.

\subsection{Composición del Comité Psicosocial}

El CPS debe estar formado preferiblemente por profesionales de diferentes áreas de conocimiento, entre las que destacamos: Psicología Clínica, Psiquiatría, Trabajo Social, Enfermería Clínica Oncológica y de Cuidados Paliativos, y Medicina, con una formación mínima sobre los aspectos psicosociales que afectan a las personas enfermas de cáncer y familiares. Además, el CPS contará con el soporte de personal administrativo. 


\subsection{Funciones y Ámbitos de las diferentes disciplinas participantes en el Comité Psicosocial}

A continuación (Tabla 2), resumimos las funciones y ámbitos de los diferentes componentes y disciplinas que participan en el Comité Psicosocial (CPS), (adaptado de Gil et al., 2020) ${ }^{(5)}$.

Tabla 2. Funciones y Ámbitos de los diferentes componentes y disciplinas participantes en el Comité Psicosocial (CPS) (adaptado de Gil et al., 2020) ${ }^{(5)}$.

Funciones y Ámbitos de los diferentes componentes y disciplinas participantes en el Comité Psicosocial (CPS)

\section{Coordinador/a}

- Organización de la presentación, discusión y asistencia de los casos presentados, así como del funcionamiento global del CPS.

- $\quad$ Organización de la formación continuada dentro del CPS.

- Establecimiento de contactos y coordinación con otros recursos psicosociales del ámbito sanitario y comunitario diferentes de los del ICO.

- Facilitación y promoción de investigación psicosocial interdisciplinaria.

\section{Psicooncólogo/a}

- Consultor/a en temas de evaluación, diagnóstico, prevención y tratamiento de los trastornos mentales, del comportamiento, emocionales, cognitivos, y relacionales necesarios para mejorar la comprensión y el abordaje de las repercusiones psicológicas que la enfermedad oncológica y sus tratamientos tienen en las personas y en la evolución misma de estas enfermedades.

\section{Psiquiatra}

- Consultor/a en temas de salud mental en el ámbito oncológico y/o cuando se requiere un abordaje psicofarmacológico; evaluación diagnóstico y tratamiento.

\section{Trabajador/a Social}

- Detección de aquellas situaciones sociofamiliares que presentan dificultades desde de la detección de la enfermedad oncológica y que están incidiendo a lo largo del proceso de la enfermedad.

- Identificación de los elementos necesarios relacionados con el enfermo y/o la familia para poder hacer frente a su situación y que resultan imprescindibles para la elaboración de un plan individualizado de trabajo.

- Establecimiento de un plan de trabajo donde aparezcan los recursos de los que se pueden beneficiar las personas con enfermedad oncológica y las familias, estableciendo un vínculo y una correcta derivación.

- Realización de intervenciones sociofamiliares que reduzcan el impacto sociofamiliar de las personas con enfermedad oncológica. 


\section{Enfermería de Práctica Avanzada Onco-hematológica y Cuidados Paliativos}

- Cribado y evaluación psicosocial de los casos que presenten en el CPS.

- Informar al resto de sus equipos o Unidades Funcionales de los casos presentados y de las decisiones tomadas en el CPS.

- Asesorar a sus equipos o Unidades Funcionales sobre evaluación y manejo psicosocial de la persona con enfermedad oncológica y sobre el funcionamiento del CPS.

- Intervenciones de educación sanitaria y Counselling en paciente y/o su familia.

- Asesoramiento en el CPS sobre el proceso oncológico (estadio, evolución, pronóstico...) y la información/comunicación con los equipos, paciente y familia para adaptar las intervenciones psicosociales.

\section{Administrativo/a}

- Programación de las visitas (primeras y sucesivas individuales o grupales) una vez se ha presentado y discutido el caso en el comité, y el/la coordinador/a haya revisado, orientado y establecido la prioridad de intervención.

- Contactar con el paciente para la persona para informarle.

- Gestión de la asistencia y Acreditación de la Formación Continuada dependiendo del CPS.

- Gestión de la programación y logística de los programas de atención psicosocial grupales.

\section{Consultores/as}

- Revisión de la organización del CPS.

- Introducción de mejoras en lo CPS.

- $\quad$ Supervisión de la formación de los miembros del CPS.

\subsection{Criterios de Derivación y Niveles de Complejidad en el CPS}

El CPS establece unos criterios de derivación y priorización de los casos que le sean remitidos. De acuerdo con estos criterios, se discuten los casos en sesión multi e interdisciplinaria, se favorece un mejor manejo psicosocial, una correcta derivación al profesional encargado con esto, una mejor gestión de los recursos asistenciales psicosociales del hospital.

\section{Criterios de derivación}

La derivación de un caso al CPS se hará de acuerdo con una evaluación psicosocial recomendada.

\section{Evaluación psicosocial recomendada}

La evaluación psicosocial que recomendamos consiste en la realización de una anamnesis breve especialmente dirigida a la detección del malestar emocional de la persona con enfermedad oncológica (o familiar) e identificación de indicadores de vulnerabilidad psicosocial. La recogida de datos se hará mediante una hoja resumen (acta) para la presentación del caso al CPS, como se puede ver en la Figura 3. 
Figura 3. Acta del Comité Psicosocial para la presentación de casos, versión 01/21 (modificado de Gil et al., 2020) ${ }^{(5)}$.

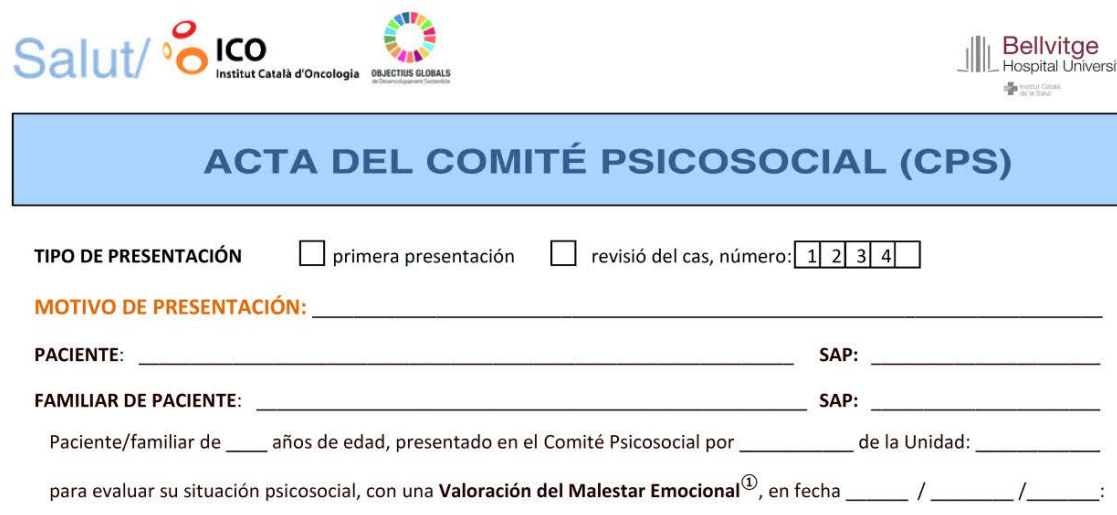

$\begin{array}{ccccccccc}\mathbf{0} & \mathbf{1} & \mathbf{2}(2,5) 3 & 4 & 5 & 6 & 7(7,5) 8 & 9 & 10 \\ \text { Nada } & & \text { Leve } & & \text { Moderado } & \text { Alto } & & \text { Máximo }\end{array} \geq 5 \quad \square$ No Procede

Datos personales (ver anexo para completar):

Estado civil:

Profesión:

Núcleo de convivencia:

Historia oncológica

Fecha del diagnóstico:

Localización:

Fase del proceso:

Número de hijos:

Situación laboral:

Hijos menores a cargo:

Cuidador principal:

Estudios:

Otro cuidador:

Criterios de presentación por complejidad (ver anexo para ampliar)

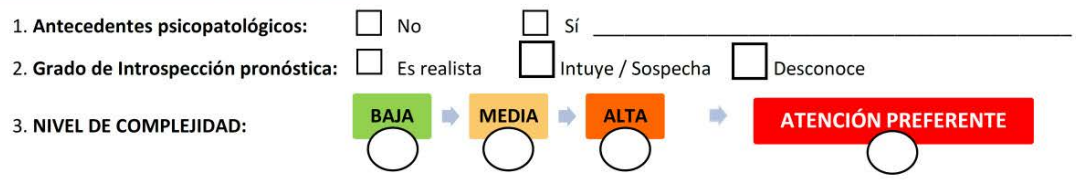

Indicadores socio-familiares

\begin{tabular}{|c|c|c|c|}
\hline Cuidador: & Sin cuidador & $\begin{array}{l}\text { Cuidador frágil o con } \\
\text { limitaciones }\end{array}$ & Sobrecarga del cuidador \\
\hline Dinámicas familiares: & $\begin{array}{l}\text { Alteración dinámicas } \\
\text { comunicación }\end{array}$ & Cambios de rol & $\begin{array}{l}\text { Dificultades en las } \\
\text { relaciones familiares }\end{array}$ \\
\hline Aspectos prácticos: & $\begin{array}{l}\text { Falta de recursos } \\
\text { material y/o prácticos }\end{array}$ & $\begin{array}{l}\text { Situación económica } \\
\text { compleja }\end{array}$ & $\begin{array}{l}\text { Necesidad de orientación y } \\
\text { asesoramiento }\end{array}$ \\
\hline $\begin{array}{l}\text { Paciente con miembros } \\
\text { vulnerables a su cargo: }\end{array}$ & SI & NO & \\
\hline $\begin{array}{l}\text { Dificultades propias de la } \\
\text { situación de inmigración: }\end{array}$ & SI & NO & \\
\hline $\begin{array}{l}\text { Dif. propias del enfermo } \\
\text { desplazado / fuera de zona: }\end{array}$ & SI & $7 \mathrm{NO}$ & \\
\hline
\end{tabular}

\section{VALORACIÓN DEL COMITÉ PSICOSOCIAL (responsables):}

Derivación PSO: Derivación PSQ: Derivación TSO: Derivación Otros: Revisión: ATENCION PREFERENTE: $\square$ No $\square$ Si

Para facilitar la cumplimentación de los datos del acta, se han añadido al final del mismo un par de anexos con información específica y más explícita y explicativa de los apartados del acta (Figuras 4 y 5). 
Figura 4. Anexo I del Acta del Comité Psicosocial, versión 01/21.

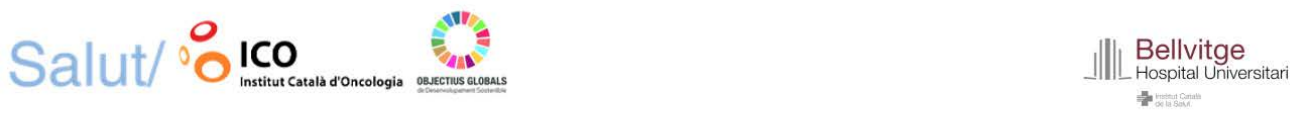

\section{ACTA DEL COMITÈ PSICOSOCIAL (CPS)}

\section{ANEXO I}

VALORACIÓN DEL MALESTAR EMOCIONAL (ME)

\begin{tabular}{|c|c|c|c|c|c|c|c|}
\hline $\mathbf{0}$ & 1 & $\mathbf{2}_{(2,5)} \mathbf{3}$ & 4 & 5 & $\boldsymbol{7}_{(7,5)} \boldsymbol{8}$ & 9 & 10 \\
\hline Nada & & Leve & & loderado & Alto & & Máximo \\
\hline 0 & & 1 & & 2 & 3 & & 4 \\
\hline
\end{tabular}

\section{DATOS PERSONALES}

- ESTADO CIVIL: $\square$ SOLTERO/A // $\square$ CASADO/A // $\square$ VIUDO/A // $\square$ DIVORCIADO/A // $\square$ SEPARADO/A // $\square$ EN PAREJA

- SITUACIÓN LABORAL:

$\square$ TRABAJADOR/A POR CUENTA AJENA

$\square$ TRABAJADOR/A POR CUENTA PROPIA (AUTÓNOMO/A)

$\square$ PARADO/A

$\square \quad$ ILT (INCAPACIDAD LABORAL TEMPORAL)

$\square$ PENSIONISTA (INVALIDEZ)

$\square$ ESTUDIANTE

$\square$ NO INFORMADA

- ESTUDIOS:

$\square$ SIN ESTUDIOS

$\square$ ESTUDIOS BÁSICOS

$\square$ ENSEÑANZA SECUNDARIA

$\square$ ENSENÑANZA PROFESIONAL

$\square$ ESTUDIOS UNIVERSITARIOS

$\square$ NO INFORMADOS

NÚCLEO DE CONVIVENCIA: $\square$ FAMILIA // $\square$ SOLO/A // $\square$ OTROS

CUIDADOR PRINCIPAL:

$\square$ SIN CUIDADOR

$\square$ FAMILIA $\rightarrow$ VER TABLA “ANTECEDENTES FAMILIARES ONCOLÓGICOS"

$\square$ OTROS CUIDADORES INFORMALES

$\square$ CUIDADOR PROFESIONAL

OTRO CUIDADOR $\rightarrow$ ÍDEM “CUIDADOR PRINCIPAL”

\section{FASE DEL PROCESO:}

\begin{tabular}{|l|l|l|l|}
\hline DIAGNÓSTICO & EN TRATAMIENTO CURATIVO / RADICAL & ENFERMEDAD AVANZADA & FINAL DE VIDA \\
\hline SUPERVIVIENTE & CIRUGÍA & PENDIENTE INICIO DE TRATAMIENTO & OTROS \\
\hline
\end{tabular}

\section{TRATAMIENTO ACTUAL:}

- EN CURSO DE TRATAMIENTO ACTIVO:

$\rightarrow \square$ QMT / $\square$ RTP / $\square$ HMT / $\square$ BQT / $\square$ INMUNOTERAPIA / $\square$ INMUNOQUIMIOT. / $\square$ TRAT. ORAL DIRIGIDO

- EN SEGUIMIENTO

CIRUGÍA

PENDIENTE DE INICIO

FUERA DE OPCIONES ONCO/HEMATO-ESPECÍFICAS (EN TRATAMIENT EXCLUSIVO PALIATIVO / SINTOMÁTICO)

NO EN TRATAMIENTO ACTIVO (LIBRE DE ENFERMEDAD, SUPERVIVIENTE, FAMILIAR DE PACIENTE) 
Figura 5. Anexo II del Acta del Comité Psicosocial, versión 01/21.

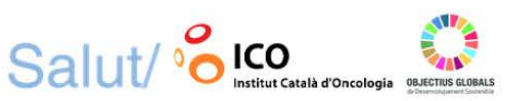

\section{ANEXO II}

\section{ANTECEDENTES PSICOPATOLÓGICOS:}

\begin{tabular}{|l|l|l|l|l|l|}
\hline & T. DEPRESIVOS & & T. ANSIEDAD & & \multicolumn{2}{|l|}{ T. ADAPTATIVOS } \\
\hline & T. PSICÓTICOS & & T. COGNOSCITIVOS & & OTROS: \\
\hline
\end{tabular}

\section{ANTECEDENTES FAMILIARES ONCOLÓGICOS:}

\begin{tabular}{|c|c|c|c|c|c|c|}
\hline \multicolumn{6}{|c|}{ GRADOS DE CONSANGUINIDAD Y AFINIDAD } & \multirow{4}{*}{ NO INFORMADOS } \\
\hline 19 Grado & PADRES & HIJOS & CÓNYUGES & SUEGROS & YERNO/NUERA & \\
\hline $2 \%$ Grado & ABUELOS & NIETOS & HERMANOS & CUÑADOS & ABUELOS CÓNYUGE & \\
\hline $3 \%$ Grado & BISABUELOS & Tíos & SOBRINOS & BIZNIETOS & & \\
\hline $4^{\circ}$ Grado & PRIMOS & SOBRINOS-NIETOS & TÍOS ABUELOS & & & NO PROCEDE \\
\hline
\end{tabular}

\section{VALORACIÓN DEL GRADO DE INTROSPECCIÓN PRONÓSTICA}

¿Qué grado de introspección crees que tiene el paciente sobre su pronóstico?

$\square$ Es Realista // $\square$ Intuye / Sospecha // $\square$ Desconoce

NIVEL DE COMPLEJIDAD (Indicadores de Vulnerabilidad / Riesgo psicosocial)

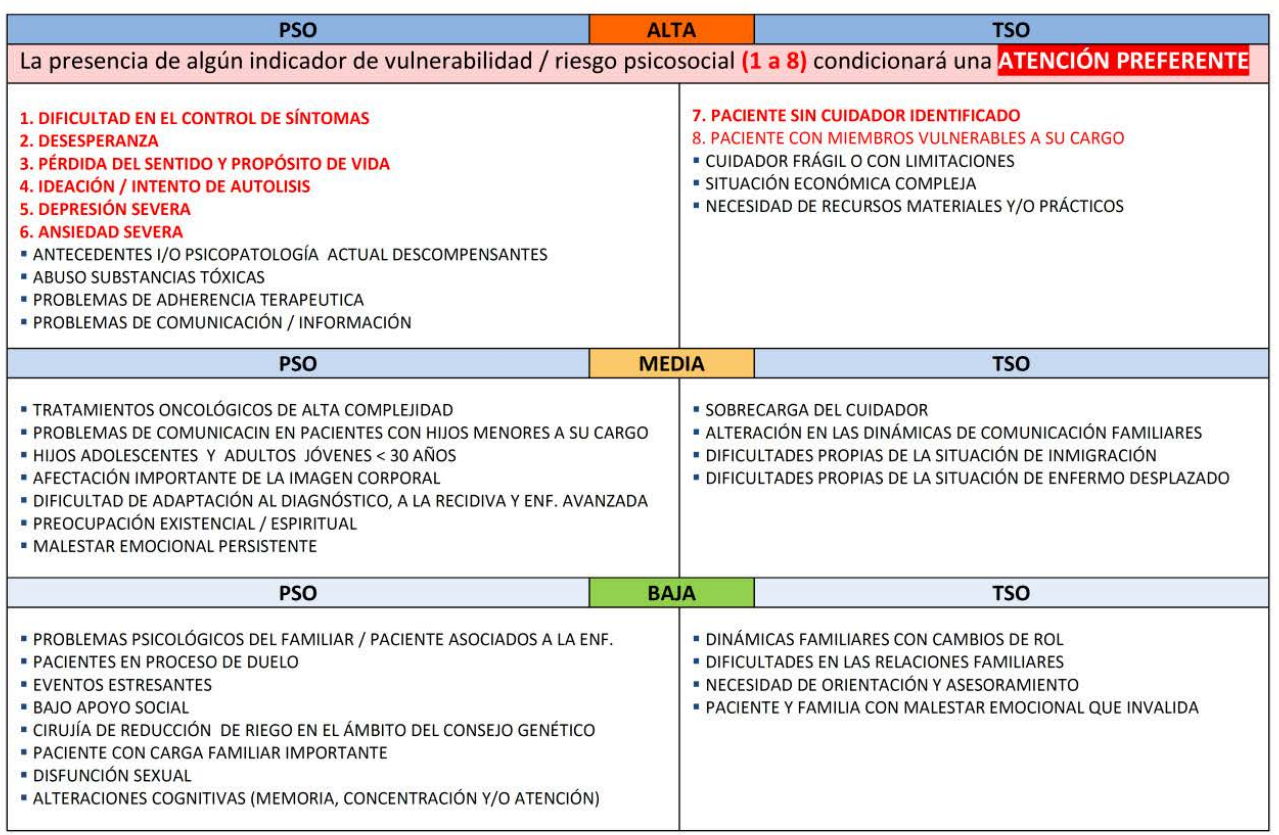




\section{Priorización por niveles de complejidad}

Hasta julio de 2018, la presentación de los casos al CPS se han ordenado según cuatro niveles de complejidad psicosocial preestablecidos (baja, media, alta y de urgencia psicosocial). Los casos que no suponían un nivel de complejidad establecido tenían que ser directamente remitidos al recurso pertinente sin pasar por el CPS (Tabla 3).

Tabla 3. Niveles de complejidad psicosocial y acciones del CPS, versión hasta 07/18.

\begin{tabular}{|l|l|}
\hline Niveles de Complejidad & Acciones del Comité Piscosocial \\
\hline Nivel I: Baja & Asesoramiento psicosocial básico para el manejo del caso. \\
\hline Nivel II: Media & $\begin{array}{l}\text { Ordenamiento, continuidad y coordinación de las } \\
\text { intervenciones psicosociales. }\end{array}$ \\
\hline Nivel III: Alta & $\begin{array}{l}\text { Se necesita una valoración interdisciplinaria consensuada } \\
\text { para intervenir. }\end{array}$ \\
\hline $\begin{array}{l}\text { Nivel IV: } \text { Alta } \rightarrow \\
\text { Urgencia Psicosocial }\end{array}$ & $\begin{array}{l}\text { Casos de intervención psicosocial urgente por la gravedad de } \\
\text { la situación psicosocial o proceso oncológico. }\end{array}$ \\
\hline
\end{tabular}

\section{Priorización por niveles de complejidad de acuerdo con la actualización a partir de un análisis con metodología Delphi $(2020)^{(4)}$.}

El estudio Delphi ${ }^{(4)}$ realizado dio como resultado unos indicadores de alta complejidad que presentamos a continuación, ordenados en cuatro áreas, 1) Médica-Física; 2) Sociofamiliar; 3) Psicoemocional; y 4) Espiritual:

1) Área Médica-Física:

a. No adherencia a los tratamientos por causa emocional (ej. Miedo a los efectos secundarios).

b. Dificultad en el control de síntomas (ej. Control del dolor).

c. Pérdida de autonomía o aumento de dependencia.

2) Área Sociofamiliar:

a. Ausencia de un entorno cuidador.

b. Cuidador/a frágil.

c. Edad joven.

d. Paciente con familiares vulnerables a su cargo.

e. Claudicación familiar.

f. Pacto de silencio a nivel familiar y del entorno de la persona con diagnóstico de cáncer.

g. Barreras impuestas por la familia.

h. Situación económica compleja con necesidades no resueltas.

3) Área Psicoemocional:

a. En consejo genético, mostrar ansiedad severa al riesgo de desarrollar cáncer. 
b. Tratamiento psiquiátrico activo.

c. Ansiedad severa.

d. Depresión severa.

e. Desesperanza.

f. Paciente hostil.

g. Ideación suicida.

h. Pensamientos recurrentes sobre la muerte.

4) Área Espiritual.

a. Pérdida de sentido y propósito de vida.

b. Deseo de adelantar la muerte.

Tras el análisis de resultados, se propuso una versión reducida de 8 criterios de alta complejidad psicosocial. En esta versión, la presencia de uno o más de estos ocho, sería razón suficiente para una derivación y atención preferente por profesionales del Comité Psicosocial (Psicooncología, Psiquiatría o Trabajo Social): ver Tabla 4.

Tabla 4. Criterios de alta complejidad psicosocial para una atención preferente por el Comité Psicosocial (CPS), (adaptado de Casellas et al., 2020)4.

La presencia de algun indicador de Vulnerabilidad / Riesgo psicosocial (1 a 8) condicionará una atención preferente

\begin{tabular}{|rl|l|}
\hline 1. & Dificultad en el control de síntomas (ej. dolor). & Área Médica-Física \\
\hline 2. & Ausencia de un entorno cuidador. & Área Sociofamiliar \\
\hline 3. & Paciente con familiares vulnerables a su cargo. & \\
\hline 4. & Ansiedad severa. & \multirow{2}{*}{ Área Psicoemocional } \\
\hline 5. & Depresión severa. & \\
\hline 6. & Desesperanza. & Área Espiritual \\
\hline 7. & Ideación suicida. & \\
\cline { 1 - 2 } 8. & Pérdida de sentido y propósito de vida. & \\
\hline
\end{tabular}

\subsection{Procedimiento}

El CPS dispone de dos tipos de sesiones con funciones diferenciadas:

Evaluación, diagnóstico y toma inicial de decisiones: sesiones que se realizan con frecuencia semanal en las que se presentan los nuevos casos. En las mismas se realiza un diagnóstico psicosocial; se establece una complejidad y prioridad 
del caso y se decide el programa terapéutico psicosocial a implementar. En esta sesión también se decide si el caso será revisado en próximas reuniones del CPS. En estas sesiones de evaluación participan todos los miembros del CPS y otros y otras profesionales sanitarios cuando tienen que presentar un caso.

- Metodología: Las personas participantes de esta sesión se reunirán durante unos 60 minutos aproximadamente. Los casos a presentar en la sesión del CPS se comunicarán telemáticamente durante los días previos a la administrativa del área de Consultas Externas encargada del CPS para que de esta manera pueda presentar el listado de los mismos a todos los miembros del CPS.

Se dispondrán de 15 minutos de media para la presentación de cada caso y la toma de decisión sobre la atención psicosocial a realizar. En total de media se presentarán de 3-5 casos, disponiendo de 15 minutos al final de la reunión para aclaraciones e informaciones de relevancia para los miembros del CPS. Una vez presentado el caso (paciente o familiar), los miembros del CPS, atendiendo a las razones por las cuales es presentado el caso, decidirán sobre la atención psicosocial que tienen que recibir. Las propuestas de atención psicosocial que se decidan no son únicas, sino que la persona podrá beneficiarse de la atención combinada de varios profesionales o programas psicosociales. Los programas psicosociales establecidos en la actualidad donde podrán ser remitidos los casos presentados, se encuentran reflejados en la Tabla 5 .

Tabla 5. Programas y Recursos de Atención Psicosocial donde puede remitir el Comité Psicosocial (CPS) tras la presentación del caso, (adaptado de Gil et al., 2004) ${ }^{(6)}$.

\section{PROGRAMAS PSICOSOCIALES ICO}

- Psicooncología (PSO).

- Psiquiatría-ICO L’H (agendas de consulta externa general, y de adicciones).

- Proyectos de investigación en atención psicosocial.

- Programa Supervivientes: comunidad virtual, grupos de terapia presencial y online.

- Grupos de soporte en enfermedad avanzada.

- Programa de atención al duelo (PAD).

- Trabajo Social Sanitario (TSO).

- Protocolo de atención a paciente desplazado.

- Protocolo de atención a paciente inmigrante.

- Enfermería Oncológica (Counselling y educación sanitaria).

- Especialidad médica referente.

\section{PROGRAMAS Y RECURSOS PSICOSOCIALES COMUNITARIOS}

- Atención Primaria (AP).

- Servicios Sociales de zona.

- Programas y Asociaciones contra el cáncer.

- Grupos de ayuda mutua.

- Grupos de acompañamiento al duelo. 
Se dejará constancia en la historia clínica las decisiones tomadas para cada uno de los casos presentados mediante el documento o acta de reunión del CPS, que llenará el personal de administración responsable y revisará la persona coordinadora del CPS. En general, la persona (paciente o familiar) podrá ser informado telefónicamente por el personal de administración del comité de las decisiones tomadas cuando la decisión sólo comporte informar de la programación de visitas que se deriven de la decisión. En los casos con mayor nivel de complejidad será el CPS quién decidirá: 1) si es necesario que la persona sea informada personalmente o telefónicamente cuando acabe la reunión donde se discute su caso; 2) si hace falta que sea un miembro del CPS quién informe o lo puede hacer el personal administrativo; y 3) quién sería el/la profesional más adecuado/a para comunicar la decisión tomada.

Las unidades y servicios del área propiamente psicosocial (Psicooncología, Trabajo Social y Psiquiatría) darán prioridad en sus agendas de Consultas Externas para una atención precoz.

Seguimiento, atención continuada y revisión de casos: son los últimos miércoles de cada mes. Corresponde a las sesiones dedicadas a revisar algunos de los casos ya presentados a las sesiones de evaluación y diagnóstico. Los miembros del CPS pueden decidir ya en las mismas reuniones de evaluación y diagnóstico si el caso será revisado, y con qué tiempo de margen se hará. Por otro lado, también será posible revisar casos que no se habían contemplado inicialmente como tributarios de revisión cuando se hayan producido cambios que lo hagan recomendable. La metodología de estas sesiones será la misma que las de presentaciones de casos nuevos.

\section{6. Índice de productividad de Psicooncología}

El presente índice recoge lo que los/las profesionales que componen este grupo de consenso consideran que debe ser el mínimo de atención psicológica para paciente y familia en consultas externas e interconsultas de planta. Los resultados de este documento surgen de un cuestionario que fue enviado a todos los Psicooncólogos del ICO con el fin de estandarizar la actividad asistencial necesaria.

A continuación, exponemos los resultados de cada una de las áreas de atención psicooncológica, incluyendo la atención en consultas externas, las interconsultas de pacientes ingresados en planta, las sesiones clínicas, la atención grupal, la atención familiar, la docencia y la investigación (Tabla 6).

En relación a la atención en consultas externas, las personas que conforman el grupo de consenso consideran que un/a profesional de la psicooncología debe realizar 5 primeras visitas semanales de una hora de duración. El tiempo de espera para una primera visita no debería ser superior a dos semanas. En lo que se refiere a las visitas sucesivas, deben realizarse 10-12 visitas sucesivas semanales de 45 minutos de duración por profesional. Cada paciente debería recibir, al menos, una visita sucesiva cada dos semanas. En total, cada profesional de la psicooncología debe distribuirse su actividad asistencial en consultas externas entre 2 ó 3 días. Como parte de la actividad asistencial, realizan informes clínicos de primeras visitas, cursos clínicos de visitas sucesivas, además de una media de dos informes externos solicitados por pacientes. La redacción de cada informe externo supone 30 minutos.

En lo que hace referencia a las interconsultas de planta, el grupo de consenso acuerda que es necesario dedicar dos días por semana para la atención a pacientes ingresados, atendiendo a un número de entre 8 y 10 pacientes por semana. Cada paciente debería 
recibir al menos dos visitas semanales, de unos 30 minutos de duración, adaptables según las necesidades de la persona. El tiempo de espera para realizar la visita no debe ser superior a 48 horas. Además de la actividad asistencial, los/as profesionales dedican parte de su actividad a la redacción de informes y cursos clínicos. Acuerdan que cada semana realizan unos 8-10 informes de pacientes ingresados de primera visita, que conllevan 30 minutos por informe. Además, realizan una media de 10 cursos clínicos semanales de visitas sucesivas, representando cada curso 15 minutos de dedicación.

Asimismo, los/las profesionales asisten al menos a una sesión de Comité Psicosocial y/o sesión clínica o de objetivos semanal de pacientes de consultas externas, de una duración de 45 minutos. Además, realizan entre una y tres sesiones clínicas o de objetivos de pacientes en procesos de hospitalización, de 30 minutos de duración.

En lo que se refiere a la atención psicológica grupal, es de dos tipos: psicoeducativa o psicoterapéutica. La frecuencia debería ser semanal, de entre 8 y 12 sesiones por grupo, de una duración de 90 minutos por sesión, más 1-2 visitas de seguimiento grupal.

El grupo de consenso acuerda la necesidad de realizar atención psicológica a la familia en planta, con sesiones de 30 minutos, y una frecuencia semanal, o de más de un día si así lo requiriese.

En lo que se refiere a la docencia e investigación debería formar parte de la actividad de los/las profesionales de la psicooncología, siendo recomendable la dedicación de un día por semana.

Pretendemos a través de estas recomendaciones de atención psicooncológica consensuadas, ayudar a determinar el índice de productividad que cada psicooncólogo/a o Unidad de Psicooncología debe tener, de acuerdo a los recursos asistenciales que dispongan (número de horas contratadas y disponibilidad de despachos). A partir de aquí, se podrá realizar el contrato de gestión (Dirección por Objetivos) con el responsable correspondiente y la Dirección de centro.

Con el fin de determinar unos mínimos que deben ser considerados dentro del índice de productividad del/de la profesional (psicooncología), recomendamos los siguientes:

- La jornada laboral semanal del/de la psicooncólogo/a será de un 80\% asistencial, y un $20 \%$ para tareas docentes y de investigación.

- Dentro de las tareas asistenciales se incluirán: visitas a paciente y familia, redacción de informes y cursos clínicos, así como la asistencia a sesiones clínicas, de objetivos o de comités asistenciales.

- La distribución de las horas de actividad asistencial, a nivel de consultas externas (primeras y sucesivas), y de interconsultas de planta (primeras y sucesivas) dependerá de la necesidad asistencial de cada centro, del número de profesionales de la psicooncologí y de los recursos (ej. número de despachos) del que disponga el centro.

- Cada centro y servicio decidirá cómo utilizar las horas dedicadas a actividad asistencial, ya sea de consultas externas o interconsultas de planta, tanto de primeras visitas como sucesivas.

- Las actividades docentes fuera del centro de trabajo estarán aprobadas por la persona responsable del Servicio y Dirección de Centro.

- Las actividades de investigación estarán acordadas con el responsable del Servicio al que pertenezca el/la profesional de la psicooncología.

- En la actualidad se ha de adaptar esta actividad cuando se realiza en formato telemático, muy relevante en la actualidad condicionado por la actual 
pandemia asociada al virus SARS-CoV-2 ${ }^{(29)}$, que se remonta desde finales del 2019. Las visitas telefónicas suelen requerir un 30-40\% menos de tiempo de media que la presencial. Las visitas realizadas por videoconferencia tanto individuales como grupales requieren aproximadamente del mismo tiempo que las presenciales. Sin embargo, queda por incluir y dimensionar correctamente dentro del tiempo asistencial otra comunicación "online" como la mensajería asincrónica y directa con pacientes y la participación en comunidades o programas de soporte online.

Asimismo, el presente documento propone unos mínimos asistenciales dentro de la cartera de servicio de una Unidad de Psicooncología. La unidad debe atender a los casos que reúnan una de las siguientes condiciones:

- Paciente en fase aguda, en tratamiento activo o paliativo.

- Supervivientes de cáncer durante el periodo necesario hasta lograr normalizar su estado emocional.

- La atención a la familia durante el periodo que dure el tratamiento activo o paliativo de la persona con diagnóstico de cáncer.

- La atención a pacientes en procesos de hospitalización y a su familia.

- Asimismo, cada unidad de psicooncología tomará las decisiones oportunas de incorporar otros programas asistenciales (duelo, consejo genético, trasplante, etc.), que consideren que deben estar dentro de la cartera de servicios de su centro.

El fin de la atención psicooncológica a paciente y familia estará determinado por la desaparición del malestar emocional significativo (síntomas de ansiedad y depresión, principalmente), y el cambio de narrativa de paciente o familiar, donde la centralidad o el foco no sea solo oncológico, y la persona dé muestras de haber asimilado e integrado la experiencia de la enfermedad, dando signos de normalización.

Tabla 6. Índice de productividad de Psicooncología.

\section{Índice de productividad de Psicooncología}

Jornada laboral (semanal)

- $80 \%$ Asistencia.

- 20\% Docencia e Investigación.

\section{Consultas Externas (atención ambulatoria)}

- Días de asistencia en consultes externes: 2-3 días por semana.

- PV: 5 (1hora/visita) por semana, TE: $\leq 2$ semanas.

- VS: 10-12 (45 min./visita/bisemanal) por semana.

- Redacción de informes clínicos (PV) y cursos clínicos (VS).

- Informes Clínicos Externos: 2/30 min./informe.

- Asistencia: Comité Psicosocial/Sesión Clínica o de Objetivos: 1 sesión/45 min./semanal. 


\section{Interconsultas (atención de pacientes en procesos de hospitalización)}

- Días de asistencia en planta: 2 días.

- $\mathrm{N}^{\mathrm{o}}$ de pacientes visitados (semanal): 8-10 (PV).

- Visitas sucesivas/paciente: 2, $30 \mathrm{~min}$./visita (adaptado a las necesidades).

- TE: $\leq 48$ horas.

- Redacción de informes (PV): 8-10 informes (30 min./informe), y cursos clínicos (VS): 10 (15 min./curso) por semana.

- Atención psicológica a la familia: 1 sesión/semanal (30 min./sesión).

- Asistencia: sesiones clínicas/objetivos: 1-3 sesiones (30 min./sesión) semanales.

\section{Atención Grupal}

Tipos: psicoeducativa y psicoterapéutica.

- $\mathrm{N}^{\mathrm{o}}$ sesiones/grupo: 8-12 sesiones

- Duración: 90 minutos.

- 1-2 visita de seguimiento grupal.

\section{Docencia e Investigación}

1 día/semanal.

Notas: PV (primera visita), VS (visita sucesiva), TE (tiempo de espera).

\section{Conclusiones}

- Este documento resumen sobre el estándar de atención psicosocial en psicooncología recoge la experiencia asistencial del grupo de profesionales y los resultados de los estudios realizados dirigidos a la mejora de la atención psicosocial en cáncer.

- La atención psicooncológica en los hospitales tiene un carácter estructural; debe formar parte de la estructura asistencial de centro hospitalario, considerando a los y las profesionales de la psicooncología como facultativos y facultativas del centro donde trabajen.

- El modelo consensuado aquí propuesto puede adaptarse a las características y recursos de cada institución. Dependiendo de estos recursos, cada centro debe decidir cuál es el mínimo asistencial que debe recoger su cartera de servicios, y cuáles deben ser los recursos asistenciales psicosociales que deben ser considerados desde la comunidad para sus pacientes y familiares.

- Esta propuesta pretende organizar la atención oncológica mediante una comunicación fluida, una coordinación lógica y eficaz entre profesionales de cada disciplina, en la misma línea de lo sugerido por Haward $(2008)^{(30)}$.

- El CPS permite una atención preferente en función de la presencia de alguno de los ocho criterios de alta complejidad definidos tras el estudio Delphi ${ }^{(4)}$ : 1) dificultades en el control de síntomas; 2 ) ausencia de un entorno cuidador; 3) paciente con familiares vulnerables a su cargo; 4) ansiedad severa; 5) depresión severa; 6) desesperanza; 7) ideación suicida; y 8) pérdida de sentido y propósito de vida.

- El abordaje de la complejidad psicosocial mediante un modelo basado en criterios multi e interdisciplinarios consensuados ayuda en la toma de decisiones sobre las acciones a seguir y en la mejora del malestar emocional y complejidad de paciente y familiar. 
- El análisis de la comparación pre y post-intervención en el CPS de los 75 a los que se les hizo el seguimiento evidencia la mejora del malestar emocional y la complejidad psicosocial tras la intervención en el CPS, con una reducción de 1,85 puntos $(\mathrm{p}<0,001)$ en malestar emocional, así como una disminución del $20 \%$ de casos de alta complejidad psicosocial. Los y las pacientes tenían edades comprendidas entre 21 y 82 años (Media $=54,46 ; \mathrm{DT}=14,75)$ y fueron reclutados entre febrero de 2017 y febrero de 2021.

- La implementación del CPS en el ICO (desde el año 2002 hasta la actualidad) en un hospital monográfico de cáncer como el ICO: 1) permite a los y las profesionales disponer de un instrumento que facilita la detección de malestar emocional y complejidad psicosocial; 2) fomenta la derivación de casos (pacientes y/o familiares) de mayor complejidad que requieren una atención preferente; 3) facilita una atención precoz y especializada multidisciplinaria; y 4) permite la optimización de recursos a través de una eficacia significativa en el abordaje y resolución de casos con alta complejidad psicosocial.

- Pese a las bondades recogidas en el presente documento, presenta limitaciones que son pertinentes señalar: 1) el tamaño de la muestra es pequeño; 2) la experiencia mostrada del CPS está basada en un único centro (ICO), y por tanto, sería fundamental validar la propuesta y los beneficios psicosociales con otras de diferentes centros.

\section{Agradecimientos}

A Candela Calle Rodríguez (Directora General, Hospital Duran i Reynals, Instituto Catalán de Oncología); y Jordi Trelis Navarro (Director Asistencial Corporativo, Hospital Duran i Reynals, Instituto Catalán de Oncología) por el apoyo en la elaboración del presente proyecto.

A las administrativas Sabina Pedrola Martí (ICO-Girona); Raquel Alcalde Cambronero y Maria Luisa Serrano Romero (ICO- L'Hospitalet) por todo el soporte recibido.

A los/as participantes habituales del Comité Psicosocial del Hospital Duran i Reynals del Instituto Catalán de Oncología, y aquellas personas que han participado puntualmente en el mismo, gracias a los cuales el CPS no ha cesado su funcionamiento desde el año 2002 con la misión de atender de la mejor manera posible la complejidad psicosocial del paciente oncológico y la familia.

\section{Referencias bibliográficas}

1. Carlson LE, Bultz BD. Efficacy and medical cost offset of psychosocial interventions in cancer care: Making the case for economic analyses. Psychooncology 2004;13:837-49. doi: $10.1002 /$ pon. 832

2. Canadian Association of Psychosocial Oncology. Standards of Psychosocial Health Services for Persons with Cancer and their Families [Internet]. 2010 [citado el 5 de febrero de 2021]. Disponible en: https://www.capo.ca/resources/Documents/3.14.\%20 National\%20Psychosocial\%20Oncology\%20Standards\%20\%20.pdf 
3. Turner J, Zapart S, Pedersen K, Rankin N, Luxford K, Fletcher J. Clinical practice guidelines for the psychosocial care of adults with cancer. Psychooncology 2005;14:159 73. doi. 10.1002/pon. 897

4. Casellas-Grau A, Jordán de Luna C, Maté J, Ochoa C, Sumalla EC, Gil F. Developing a consensus definition of psychosocial complexity in cancer patients using Delphi methods. Palliat Support Care 2020; 25:1-11. doi: 10.1017/S1478951520000784

5. Gil Moncayo FL, Maté Méndez J, Ochoa Arnedo C, Casellas-Grau A, Trelis Navarro J, Borràs Andrés JM. Abordaje de la complejidad psicosocial en pacientes con cáncer. Med Clin (Barc) 2020;154:86-93. doi: 10.1016/j.medcli.2020.03.021

6. Gil F, Novellas A, Barbero E, Hollenstein MF, Maté J. Modelo de atención psico-social en oncología: Standards. Psicooncologia 2004;1:179-84.

7. Zabora, J, Brintzenhofe Szoc K, Curbow B, Hooker C, Piantadosi S. The prevalence of psychological distress by cancer site. Psychooncology 2001; 10:19-28. doi: 10.1002/1099-1611(200101/02)10:1<19::aid-pon501>3.0.co;2-6

8. Carlson LE, Angen M, Cullum J, Goodey E, Koopmans J, Lamont L, et al. High levels of untreated distress and fatigue in cancer patients. Br J Cancer 2004; 90:297-304. Doi. 10.1038/sj.bjc. 6601887

9. Mitchell AJ, Ferguson DW, Gill J, Paul J, Symonds P. Depression and anxiety in longterm cancer survivors compared with spouses and healthy controls: a systematic review and meta-analysis. Lancet Oncol 2013; 14:721-32. doi: 10.1016/S1470-2045(13)70244-4

10. Grassi L, Travado L, Gil F, Sabato S, Rossi E, SEPOS Group. Psychosocial morbidity and its correlates in cancer patients of the Mediterranean area: findings from the Southern European Psycho-Oncology Study (SEPOS). J Affective Disor 2004; 83:243-48.

11. Grassi L, Riba M. Clinical Psycho-Oncology: An International Perspective. Wiley: Chichester, 2012. doi: 10.1016/j.jad.2004.07.004

12. Grassi L, Travado L. The role of psychosocial oncology in cancer care. In: Coleman MP, Alexe DM, Albreht T, McKee M, eds. Responding to the Challenge of Cancer in Europe. Ljubljana: Institute of Public Health, 2008. p. 209-29.

13. Mehnert A, Koch U, Schulz H, Wegscheider K, Weis J, Faller H, et al. Prevalence of mental disorders, psychosocial distress and need for psychosocial support in cancer patients - study protocol of an epidemiological multi-center study. BMC Psychiatry 2012;12:70. doi: 10.1186/1471-244X-12-70

14. McCarter K, Britton B, Baker A, Halpin S, Beck A, Carter G, et al. Interventions to improve screening and appropriate referral of patients with cancer for distress: systematic review protocol. BMJ Open 2015;5:e008277. doi: 10.1136/bmjopen-2015-008277

15. Singer S, Das-Munshi J, Brähler E. Prevalence of mental health conditions in cancer patients in acute care--a meta-analysis. Ann Oncol 2010;21:925-30. doi: 10.1093/annonc/mdp515

16. Grassi L, Spiegel D, Riba M. Advancing psychosocial care in cancer patients. F1000Research 2017; 6: 2083. doi: 10.12688/f1000research.11902.1

17. Wilson KG, Chochinov HM, Skirko MG, Allard P, Chary S, Gagnon PR, et al. Depression and anxiety disorders in palliative cancer care. J Pain Symptom Manage 2007;33:118-29. doi: 10.1016/j.jpainsymman.2006.07.016

18. Mitchell AJ, Chan M, Bhatti H, Halton M, Grassi L, Johansen C, et al. Prevalence of depression, anxiety, and adjustment disorder in oncological, haematological, and palliative-care settings: a meta-analysis of 94 interview-based studies. Lancet Oncol 2011;12:160-74. doi: 10.1016/S1470-2045(11)70002-X

19. Galán M, Farran L, Aliste L, Hormigo G, Aranda H, Bettonica C, et al. Multidisciplinary cancer care may impact on the postoperative mortalityand survival of patients with 
oesophageal and oesophagogastric junction cancer: A retrospective cohort study. Clin Transl Oncol 2015;17:247-56.14. doi: 10.1007/s12094-014-1221-7

20. Temel JS, Greer JA, Muzikansky A, Gallagher ER, Admane S, Jackson VA, et al. Early palliative care for patients with metastatic non-small-cell lung cancer. N Engl J Med. 2010;363:733-42. doi: 10.1056/NEJMoa1000678

21. gencat. Instituto Catalán de Oncología. Política de calidad. Instituto Catalán de Oncología. [Internet]. 2012 [citado el 5 de febrero de 2021]. Disponible en: http://ico.gencat.cat/ es/1_institut/informacio-corporativa/politica_de_qualitat/

22. Borràs JM, Albreht T, Audisio R, Briers E, Casali P, Esperou H, et al. European Partnership Action Against Cancer consensus grou. Policy statement on multidisciplinary cancer care. Eur J Cancer 2014;50:475-80. doi: 10.1056/NEJMoa1000678

23. Borràs JM, Federation of Spanish Oncology Societies, Spanish Society of Pathology, Spanish Society of Palliative Care, Spanish Society of Oncology Nursing, Spanish Society of Haematology and Haemotherapy, et al. Institutional declaration in favour of the development of interdisciplinary cancer care in Spain. Clin Transl Oncol 2010;12:587-9. doi: 10.1007/s12094-010-0562-0.

24. Salvador MR, Garrido N, Perea I, Martín ML, Regife V, Fernández López A. (2017) El valor del Instrumento Diagnóstico de la Complejidad en Cuidados Paliativos para identificar la complejidad en pacientes tributarios de cuidados paliativos. Medicina Paliativa 24, 196-203. doi: 10.1016/j.medipa.2016.01.003

25. Tuca A, Gómez-Martínez M, Prat A. Predictive model of complexity in early palliative care: A cohort of advanced cancer patients (PALCOM study). Supportive Care in Cancer 2018;26: 241-9. doi: 10.1007/s00520-017-3840-3

26. Travado L, Dalmas M. Psychosocial Oncology Care. En: European Guide for the Quality National Cancer Control Programmes. Editado por: Albreht T, Martín-Moreno J.M., Jelenc M, Gorgojo L, Harris M. National Institute of Public Health, Ljubljana, Slovenia, 2015.

27. Travado L, Reis JC, Watson M, and Borràs JM. Psychosocial oncology care resources in Europe: a study under the European Partnership for Action Against Cancer (EPAAC). Psychooncology 2017;26:523-30. doi: 10.1002/pon.4044.

28. Maté Méndez J, Ochoa Arnedo C, Hernández Ribas R, Segalàs Cosi C. Síntomas psicológicos y psiquiátricos: Evaluación emocional básica. En: Julià-Torras J, Serrano Bermúdez G. (eds): Manual Control de síntomas en Pacientes con Cáncer. 4. ${ }^{\mathrm{a}}$ ed. Madrid: Arán, 2019. p. 263-75.

29. WHO Director-General's remarks at the media briefing on 2019-nCoV on 11 February 2020 [Internet]. [citado el 5 de febrero de 2021]. Disponible en: https:/www.who.int/ director-general/speeches/detail/who-director-general-s-remarks-at-the-media-briefingon-2019-ncov-on-11-february-2020

30. Haward R. Organising a comprehensive framework for cancer control. En: Cole-man MP, Alexe DM, Albreht T, McKee M, editores. Responding to the Challenge of Cancer Care in Europe. Ljubljana: Institute of Public Health of the Republic of Slovenia and European Observatory on Health Systems and Policies; 2008. p.113-134. 